\title{
The Edwards-Wilkinson equation with drift and Neumann boundary conditions
}

\author{
Seng Cheang and Gunnar Pruessner \\ E-mail: g.pruessner@imperial.ac.uk, seng.cheang@imperial.ac.uk \\ Department of Mathematics, Imperial College London, 180 Queen's Gate, London \\ SW7 2AZ, UK
}

\begin{abstract}
The well known scaling of the Edwards-Wilkinson equation is essentially determined by dimensional analysis. Once a drift term is added, more sophisticated reasoning is required, which initially suggests that the drift term dominates over the diffusion. However, the diffusion term is dangerously irrelevant and the resulting scaling in fact non-trivial. In the present article we compare the resulting scaling of the Edwards-Wilkinson equation with drift and Neumann boundary conditions to the published case with Dirichlet boundary conditions.
\end{abstract}

Submitted to: J. Phys. A: Math. Gen.

PACS numbers: 05.70.Np, 68.35.Ct, 68.35.Rh 


\section{Introduction}

The Edwards-Wilkinson equation [1] is very well understood. It is a stochastic equation of motion describing the most basic surface evolution of a growth model, consisting merely of diffusion and random particle deposition. Given its minimal parameterisation in terms of a diffusion constant $D$, a noise amplitude $\Gamma$ as well as the system size $L$ and time $t$, Equation (1) and (2), the scaling of its roughness in $L$ and $t$ is determined by dimensional analysis, because the only dimensionless quantity is $D t / L^{2}$. This changes, as soon as another interaction term is added whose coupling allows an additional dimensionless quantity to be formed. In principle, the scaling is then not determined by simple dimensional arguments. In this situation, it is common to invoke physical arguments to show that one of the terms is (infrared) irrelevant and thus can be dropped [2, 3, 4]. There is no mathematically rigorous argument underpinning this procedure and it therefore can produce incorrect results. This has been analysed in the past [5] for the addition of a drift (convection) term to the Edwards-Wilkinson equation, which first seems to render the diffusion irrelevant, when in fact it becomes dangerously irrelevant [6] and thus should not be dropped from the analysis.

It has been noted [5] that the rôle of the drift term depends on the boundary condition. It is trivial to show that in the presence of periodic boundaries the drift does not enter the roughness (in the definition below) at all, but changes the scaling when Dirichlet boundary conditions are applied. One might now wonder to what extend the result is universal, i.e. what happens, for example, if Neumann boundary conditions are applied. The calculations are far more involved than for the Dirichlet case and presented in the following.

It is not surprising if boundary conditions change some universal quantities at the critical point [7], certainly not if there is some net transport across the system as in the present case. Given that the system is apparently sensitive to the choice of boundary condition, the finding below might come as a surprise; Neumann and Dirichlet boundary conditions produce, in fact, the same leading order behaviour.

Beyond its rôle of probing scaling and universality in the Edwards-Wilkinson equation, the drift term has a simple physical motivation. It could, for example, be caused by a gravitational or an electric field, present during epitaxial growth. The choice of the form of the boundary condition is a bit more arbitrary. A Dirichlet boundary condition corresponds to particles falling off the edge of the substrate. At the same time, particles are to be generated whenever a hole forms at the boundary, which makes the boundary condition somewhat unphysical. As discussed below, the Neumann boundary is an improvement in this respect, but still does not correspond an easily implemented physical situation, for example by allowing no net flux across the boundary. The motivation for the choice of the Neumann boundary condition in the following is thus the question to what extent the choice of the boundary condition changes the universal behaviour of the system compared to the Dirichlet case. 


\subsection{Preliminaries}

The features of the original Edwards-Wilkinson (EW) equation [1]

$$
\partial_{t} \phi(\mathbf{x}, t)=D \nabla^{2} \phi(\mathbf{x}, t)+\eta(\mathbf{x}, t)
$$

have been reviewed a number of times [2, 8, 9, 10, 11. The field $\phi(\mathbf{x}, t)$ describes the height of an interface over a substrate of dimension $d$ and linear extension $L$ at position $\mathbf{x}$ and time $t$. The noise $\eta(\mathbf{x}, t)$ is Gaussian and white, characterised by the usual correlator

$$
\left\langle\eta(\mathbf{x}, t) \eta\left(\mathbf{x}^{\prime}, t^{\prime}\right)\right\rangle=\Gamma^{2} \delta\left(\mathbf{x}-\mathbf{x}^{\prime}\right) \delta\left(t-t^{\prime}\right)
$$

with amplitude $\Gamma^{2}$ and $\langle\eta\rangle=0$. The operation $\langle\cdot\rangle$ describes the averaging over the noise, such that, for example, $\partial_{t}\langle\phi(\mathbf{x}, t)\rangle=D \nabla^{2}\langle\phi(\mathbf{x}, t)\rangle$. In the context of growth phenomena, the observable of choice is often the roughness, which is the ensemble average of the spatial variance of the field $\phi(\mathbf{x}, t)$

$$
w^{2}(L, t)=\left\langle\overline{\phi(\mathbf{x}, t)^{2}}-\overline{\phi(\mathbf{x}, t)}^{2}\right\rangle
$$

where ${ }^{-}$describes the spatial average

$$
-=\frac{1}{L^{d}} \int_{L^{d}} \mathrm{~d}^{d} x
$$

over the entire substrate of volume $L^{d}$. The roughness is closely related to the correlation function $C\left(\mathbf{x}, \mathbf{x}^{\prime}, t\right)=\left\langle\left(\phi(\mathbf{x}, t)-\phi\left(\mathbf{x}^{\prime}, t\right)\right)^{2}\right\rangle$, as

$$
w^{2}(L, t)=\frac{1}{2} \frac{1}{L^{d}} \int_{L^{d}} \mathrm{~d}^{d} x^{\prime} \frac{1}{L^{d}} \int_{L^{d}} \mathrm{~d}^{d} x C\left(\mathbf{x}, \mathbf{x}^{\prime}, t\right) .
$$

The scaling of the roughness in time is characterised by the universal dynamical exponent $z$ and the finite size scaling in $L$ by the universal roughness exponent $\alpha$, which is summarised in the Family-Vicsek scaling hypothesis [12]

$$
w^{2}(L, t)=a L^{2 \alpha} \mathcal{G}\left(\frac{t}{b L^{z}}\right)
$$

where $a$ and $b$ are two non-universal metric factors [7]. The (universal) dimensionless scaling function $\mathcal{G}(x)$ mediates between two regimes, as $\lim _{x \rightarrow \infty} \mathcal{G}(x)=\mathcal{G}_{\infty}$ with $0<\mathcal{G}_{\infty}<\infty$ and $\mathcal{G}(x) \sim x^{2 \beta}$ for $x \rightarrow 0$ with the roughening exponent $\beta$ obeying $z \beta=\alpha$. Taking the limit $t \rightarrow \infty$ first, the roughness thus scales like $w^{2}(L, t) \propto L^{2 \alpha}$ in increasing $L$; taking the thermodynamic limit $L \rightarrow \infty$ first, it scales like $w^{2}(L, t) \propto t^{2 \beta}$ with increasing $t$. Subleading terms in $L$ and $t$ respectively are expected to be suppressed with increasing $t$ and $L$ respectively.

Given that the Edwards-Wilkinson equation in the form (1) is fully parameterised by $L, t$ and $D, \Gamma^{2}$, the only possible scaling is

$$
w^{2}\left(L, t ; D, \Gamma^{2}\right)=\frac{\Gamma^{2}}{D} L^{2-d} \mathcal{G}\left(\frac{D t}{L^{2}}\right)
$$

so that $\alpha=(2-d) / 2$ and $z=2$, unless $w^{2}$ does not exist, diverges or vanishes. In fact, at and above the upper critical dimension $d_{c}=2$, the roughness is controlled by a lower cutoff or lattice spacing $a$, and diverges for $d>d_{c}$ like $a^{2-d}$ as $a \rightarrow 0$ [8]. 
In the following, the Edwards-Wilkinson equation is studied in $d=1$ dimensions, where

$$
\alpha=1 / 2 \quad \beta=1 / 4 \quad z=2 \quad \text { (standard EW) . }
$$

Provided that no other scales are present, the result (7) from dimensional analysis determines the scaling. Boundary conditions generally affect the scaling function $\mathcal{G}(x)$, and the metric factors $a$ and $b$, (6) , but not the exponents. Some types of interactions can be added to the Edwards-Wilkinson equation without affecting its scaling behaviour, which hints at the universality alluded to earlier. This can be illustrated by adding a term $-\nu\left(\nabla^{2}\right)^{2} \phi$ on the right of Equation (1), which results in a scaling of the roughness like

$$
w^{2}\left(L, t ; D, \Gamma^{2}, \nu\right)=\frac{\Gamma^{2}}{D} L^{2-d} \mathcal{G}^{\prime}\left(\frac{D t}{L^{2}}, \frac{t \nu}{L^{4}}\right) .
$$

Irrespective of $t$, the parameter $t \nu / L^{4}$ vanishes with increasing $L$ much faster than the parameter $t D / L^{2}$, so that $w^{2}\left(L, t ; D, \Gamma^{2}, \nu\right)$ approaches $w^{2}\left(L, t ; D, \Gamma^{2}, 0\right)$, assuming $\mathcal{G}^{\prime}\left(D t / L^{2}, t \nu / L^{4}\right) \approx \mathcal{G}\left(D t / L^{2}\right)$ for sufficiently large $L$ and irrespective of $t$, in particular irrespective of whether $t$ is held fixed or the limit $t \rightarrow \infty$ is taken. This amounts to the statement that $-\nu\left(\nabla^{2}\right)^{2} \phi$ is asymptotically irrelevant, so that the scaling of the Edwards-Wilkinson equation of the original form (1) is recovered.

\section{The Edwards Wilkinson equation with drift}

Whether a term is deemed relevant is a matter of the canonical dimension of the coupling. Changing (1) to the one-dimensional Edwards-Wilkinson equation with drift (EWd),

$$
\partial_{t} \phi(x, t)=D \partial_{x}^{2} \phi(x, t)+v \partial_{x} \phi(x, t)+\eta(x, t),
$$

at first seems to suggest that $D$ is irrelevant at any finite $v$, suggesting a scaling of the form

$$
w^{2}\left(L, t ; v, \Gamma^{2}\right)=\frac{\Gamma^{2}}{v} L^{1-d} \mathcal{G}^{\prime \prime}\left(\frac{t v}{L}\right)
$$

so that

$$
\alpha=0 \quad \beta=0 \quad z=1 \quad \text { (suspected EWd) . }
$$

This result, however, is obviously flawed, if periodic boundary conditions (PBC) are applied. In that case the drift term can be transformed away by a Galilean transformation, $\tilde{\phi}(x, t)=\phi(x-v t, t)$, so that $\tilde{\phi}(x, t)$ follows the the original EW equation (11) and thus the roughness of the interface displays the scaling derived in (7),

$$
\alpha=1 / 2 \quad \beta=1 / 4 \quad z=2 \quad \text { (EWd with } \mathrm{PBC}) .
$$

Since the initial guess (12) is based on a purely physical argument (rather than a mathematical one), this result merely questions the validity of this type of reasoning. It is clear that if $v$ does not actually enter into the observable $w^{2}$ as defined in (3) because it can be transformed away, then it cannot dominate its scaling. As done below, where 
$v$ can not be transformed away, one might equally argue that $D$ never becomes truly irrelevant, i.e. the exponents (13) are a result of $D$ being dangerously irrelevant [6].

$A$ priori nothing can thus be said about the relevance of the couplings $D$ and $v$. The scaling of the roughness has to be written as

$$
w^{2}\left(L, t ; D, \Gamma^{2}, v\right)=\frac{\Gamma^{2}}{D} L \tilde{\mathcal{G}}\left(\frac{D t}{L^{2}}, \frac{v t}{L}\right)
$$

which no longer fixes the scaling exponents $\alpha, \beta$ and $z$, as defined through Equation (6) , based on a scaling function on a single argument. The problem is the appearance of the new dimensionless parameter $v t / L$, which can alter the asymptotic behaviour of $w^{2}$ in a completely unknown way. In fact, with Dirichlet boundary conditions (BC), $\phi(x=0, t)=\phi(x=L, t)=0$, it was found [5] that $\tilde{\mathcal{G}}\left(D t / L^{2}, v t / L\right)$ scales like $(D /(v L))^{1 / 2}$ as $t \rightarrow \infty$ at fixed $L$ and like $\left(D t / L^{2}\right)^{1 / 2}$ for $L \rightarrow \infty$ at fixed $t$, in summary

$$
w^{2}\left(L, t ; D, \Gamma^{2}, v\right)=\Gamma^{2} \sqrt{\frac{L}{D v}} \tilde{\mathcal{G}}^{\prime}\left(\frac{v t}{L}\right)
$$

with $\tilde{\mathcal{G}}^{\prime}(x) \rightarrow$ const. as $x \rightarrow 0$ and $\tilde{\mathcal{G}}^{\prime}(x) \propto \sqrt{x}$ as $x \rightarrow \infty$ (with corrections in powers of $\left.D t / L^{2}\right)$, so that

$$
\alpha=1 / 4 \quad \beta=1 / 4 \quad z=1 \quad(\text { EWd with Dirichlet } \mathrm{BC}) .
$$

Depending on the boundary condition, the additional scale $v$ thus either leaves the scaling of the Edwards-Wilkinson equation unchanged (as seen in the case of periodic $\mathrm{BC}$ ), or changes them to anomalous values, which cannot be recovered from a simple dimensional analysis (as in the case of Dirichlet BC). The remainder of the present article is dedicated to the question which scaling behaviour is generated by Neumann boundary conditions, $\partial_{x} \phi(x=0, t)=\partial_{x} \phi(x=L, t)=0$, which could lead, in principle, to a third set of exponents.

\subsection{Neumann boundary conditions}

If $\phi(\mathbf{x}, t)$ is the height of a grown surface at time $t$ atop a substrate at position $\mathbf{x}$, the Dirichlet boundary condition mentioned above seems slightly unphysical, because it is difficult to imagine a mechanism that would pin the height to a certain value at the boundary of the surface, by taking up any excess amount of matter and providing it when needed. A zero flux boundary condition is much more naturally implemented, as it corresponds to imposing that no matter can enter or leave the substrate at the boundaries. The flux in (10) is

$$
j(x, t)=-\left(D \partial_{x}+v\right) \phi(x, t)
$$

such that $\partial_{t} \phi=-\partial_{x} j$ as a matter of mass balance (ignoring the noise). The condition $j(x, t)=0$ at $x=0, L$ is, however, very difficult to analyse. Since the true motivation of this study is the question to what extent the Edwards-Wilkinson equation with drift displays universal behaviour, in the following the weaker Neumann condition $\partial_{x} \phi(x, t)=0$ for $x=0, L$ will be analysed. 
To ease notation, it is helpful to absorb the various couplings and other dimensionful quantities in (10) into a redefinition of time, space, field and noise. With $y=x / L \in$ $[0,1], \tau=D t / L^{2}$ and coupling $q=v L / D$ Equation (10) becomes

$$
\partial_{\tau} \varphi(y, \tau)=\partial_{y}^{2} \varphi(y, \tau)+q \varphi(y, \tau)+\xi(y, \tau)
$$

where

$$
\varphi(y, \tau)=\sqrt{\frac{D}{L \Gamma^{2}}} \phi(x, t) \text { and } \xi(y, \tau)=\sqrt{\frac{L^{3}}{D \Gamma^{2}}} \eta(x, t)
$$

so that

$$
\left\langle\xi(y, \tau) \xi\left(y^{\prime}, \tau^{\prime}\right)\right\rangle=\delta\left(y-y^{\prime}\right) \delta\left(\tau-\tau^{\prime}\right) .
$$

The Neumann boundary conditions correspond to

$$
\left.\partial_{y}\right|_{y=0,1} \varphi(y, \tau)=0 \text {. }
$$

As suggested by naive dimensional analysis, the coupling $q$ seems to play an ever increasingly important rôle, as it diverges in the thermodynamic limit $L \rightarrow \infty$. An alternative re-parameterisation of the drift that does not suffer from this problem is the dimensionless quantity $u=t v^{2} / D$, which will be of great use below.

The formal solution of Equation (18) for a given realisation of the noise $\xi(y, \tau)$

$$
\varphi(y, \tau)=\int_{0}^{\tau} \mathrm{d} \tau^{\prime} \int_{0}^{1} \mathrm{~d} y^{\prime} G\left(y, \tau-\tau^{\prime} ; y^{\prime}, q\right) \xi\left(y^{\prime}, \tau^{\prime}\right)
$$

is based on the Green function $G\left(y, \tau-\tau^{\prime} ; y^{\prime}, q\right)$ which describes the propagation of a Dirac delta peak at $y^{\prime}$ at time $\tau^{\prime}$ to $y$ at time $\tau$. It is determined by considering the deterministic, homogeneous partial differential equation (PDE)

$$
\partial_{\tau} G=\left(\partial_{y}^{2}+q \partial_{y}\right) G
$$

with initial condition $\lim _{\tau \rightarrow 0} G\left(y, \tau ; y_{0}, q\right)=\delta\left(y-y_{0}\right)$ and Neumann boundary conditions, which in turn can be constructed from a complete set of eigenfunctions of the Sturm-Liouville problem

$$
\lambda_{n} g_{n}(y)=\left(\partial_{y}^{2}+q \partial_{y}\right) g_{n}(y) .
$$

The operator can be made self-adjoint with the help of a suitable weight function, as discussed in great detail in [13], $\langle f \mid g\rangle=\int_{0}^{1} \mathrm{~d} y e^{q y} f(y) g(y)$. The set of normalised eigenfunctions orthogonal with respect to this scalar product is then found as

$$
g_{n}(y)=e^{-\frac{1}{2} q y} \sqrt{\frac{2}{k_{n}^{2}+(1 / 4) q^{2}}}\left[k_{n} \cos \left(k_{n} y\right)+\frac{1}{2} q \sin \left(k_{n} y\right)\right]
$$

and $\lambda_{n}=-(1 / 4) q^{2}-k_{n}^{2}$ for $n \geq 1$, where $k_{n}=\pi n$. The eigenfunction without a node, $n=0$, deviates from that pattern,

$$
g_{0}(y)=\sqrt{\frac{q}{e^{q}-1}}
$$

and $\lambda_{0}=0$. Having introduced a scalar product above that renders the differential operator self-adjoint, the temporal evolution of any initial distribution under the PDE 
(23) can be determined. On this basis, or equivalently, on the basis of completeness, the Green function is found to be [14, p. 63]

$$
\begin{aligned}
G\left(y, \tau ; y_{0}, q\right)= & e^{q y_{0}} \frac{q}{e^{q}-1}+e^{-\frac{1}{2} q\left(y-y_{0}\right)-\frac{1}{4} q^{2} \tau} \sum_{n=1}^{\infty} \frac{2 e^{-k_{n}^{2} \tau}}{k_{n}^{2}+(1 / 4) q^{2}} \\
& \times\left(k_{n} \cos \left(k_{n} y_{0}\right)+\frac{1}{2} q \sin \left(k_{n} y_{0}\right)\right)\left(k_{n} \cos \left(k_{n} y\right)+\frac{1}{2} q \sin \left(k_{n} y\right)\right)
\end{aligned}
$$

2.1.1. Poisson summation In principle, the Green function (27) could now be used in (22) and the asymptotic properties of the roughness (3) be determined. However, it very quickly turns out that the real space limit of large $L$ are very difficult to handle in Fourier space, $k_{n}$, and the sums appearing in (27) are practically intractable. A Poisson summation could be performed at any stage, but is most easily done directly on $G\left(y, \tau ; y_{0}, q\right)$ itself. To this end, one notes that

$$
\begin{aligned}
\frac{1}{k_{n}^{2}+(1 / 4) q^{2}} & \left(k_{n} \cos \left(k_{n} y_{0}\right)+\frac{1}{2} q \sin \left(k_{n} y_{0}\right)\right)\left(k_{n} \cos \left(k_{n} y\right)+\frac{1}{2} q \sin \left(k_{n} y\right)\right)= \\
& \frac{1}{2}\left(\cos \left(k_{n}\left(y-y_{0}\right)\right)+\cos \left(k_{n}\left(y+y_{0}\right)\right)\right) \\
& +\frac{1}{2} \frac{q}{k_{n}^{2}+(1 / 4) q^{2}}\left(k_{n} \sin \left(k_{n}\left(y+y_{0}\right)\right)-\frac{1}{2} q \cos \left(k_{n}\left(y+y_{0}\right)\right)\right),
\end{aligned}
$$

so that

$$
\begin{aligned}
G\left(y, \tau ; y_{0}, q\right)= & e^{q y_{0}} \frac{q}{e^{q}-1}+e^{-\frac{1}{2} q\left(y-y_{0}\right)-\frac{1}{4} q^{2} \tau} \\
& \times \sum_{n=1}^{\infty} e^{-k_{n}^{2} \tau}\left(\cos \left(k_{n}\left(y-y_{0}\right)\right)+\cos \left(k_{n}\left(y+y_{0}\right)\right)\right) \\
& +e^{-\frac{1}{2} q\left(y-y_{0}\right)-\frac{1}{4} q^{2} \tau} \sum_{n=1}^{\infty} \frac{q e^{-k_{n}^{2} \tau}}{k_{n}^{2}+(1 / 4) q^{2}} \\
& \times\left(k_{n} \sin \left(k_{n}\left(y+y_{0}\right)\right)-\frac{1}{2} q \cos \left(k_{n}\left(y+y_{0}\right)\right)\right) .
\end{aligned}
$$

This might not look like a significant improvement unless one notes that [15, p. 373]

$$
\theta_{3}(z, \tau)=1+2 \sum_{n=1}^{\infty} e^{\imath \pi \tau n^{2}} \cos (2 n \pi z)=\frac{1}{\sqrt{-\imath \tau}} \sum_{n=-\infty}^{\infty} e^{-\imath \frac{\pi(z+n)^{2}}{\tau}}
$$

which allows the re-summation of the two cosine terms in (29),

$$
\begin{aligned}
G\left(y, \tau ; y_{0}, q\right)= & e^{q y_{0}} \frac{q}{e^{q}-1}-e^{-\frac{1}{2} q\left(y-y_{0}\right)-\frac{1}{4} q^{2} \tau}+e^{-\frac{1}{2} q\left(y-y_{0}\right)-\frac{1}{4} q^{2} \tau} \sum_{n=1}^{\infty} \frac{q e^{-k_{n}^{2} \tau}}{k_{n}^{2}+(1 / 4) q^{2}} \\
& \times\left(k_{n} \sin \left(k_{n}\left(y+y_{0}\right)\right)-\frac{1}{2} q \cos \left(k_{n}\left(y+y_{0}\right)\right)\right)+e^{-\frac{1}{2} q\left(y-y_{0}\right)-\frac{1}{4} q^{2} \tau} \frac{1}{\sqrt{4 \pi \tau}} \\
& \times \sum_{n=-\infty}^{\infty}\left(e^{-\frac{\left(y-y_{0}+2 n\right)^{2}}{4 \tau}}+e^{-\frac{\left(y+y_{0}+2 n\right)^{2}}{4 \tau}}\right) .
\end{aligned}
$$

Without the exponential prefactor, $\exp \left[-(1 / 2) q\left(y-y_{0}\right)-(1 / 4) q^{2} \tau\right]$, the last summation describes the diffusive motion of particles with (positive) mirror image on a ring of 
circumference 2 not being subject to the drift. In the following, the aim is to express Equation (31) in terms of the free propagator on $\mathbb{R}$

$$
\Phi_{0}(y, \tau ; q)=\frac{1}{\sqrt{4 \pi \tau}} e^{-\frac{(y+q \tau)^{2}}{4 \tau}},
$$

which solves (23), so that none of the terms is expressed any longer in Fourier space, which facilitates integration and the determination of the asymptote using a saddle point approximation. In fact, including all pre-factors, the last sum in (31) can be written as

$$
\sum_{n=-\infty}^{\infty} e^{q n}\left(\Phi_{0}\left(y-y_{0}+2 n, \tau ; q\right)+e^{q y_{0}} \Phi_{0}\left(y+y_{0}+2 n, \tau ; q\right)\right) .
$$

The first three terms in (31), the two exponentials and the sum, are much more difficult to handle. Taking the limit $\tau \rightarrow 0$, the expression

$$
\begin{aligned}
s\left(y, y_{0}\right)=e^{q y_{0}} & \frac{q}{e^{q}-1}-e^{-\frac{1}{2} q\left(y-y_{0}\right)}+e^{-\frac{1}{2} q\left(y-y_{0}\right)} \sum_{n=1}^{\infty} \frac{q}{k_{n}^{2}+(1 / 4) q^{2}} \\
& \times\left(k_{n} \sin \left(k_{n}\left(y+y_{0}\right)\right)-\frac{1}{2} q \cos \left(k_{n}\left(y+y_{0}\right)\right)\right) \\
& =\frac{q e^{q y_{0}}}{e^{q}-1}+\frac{1}{2} q e^{-\frac{1}{2} q\left(y-y_{0}\right)} \sum_{n=-\infty}^{\infty} \frac{1}{k_{n}^{2}+(1 / 4) q^{2}} \\
& \times\left(k_{n} \sin \left(k_{n}\left(y+y_{0}\right)\right)-\frac{1}{2} q \cos \left(k_{n}\left(y+y_{0}\right)\right)\right)
\end{aligned}
$$

can be regarded as an initial source which is propagated under the PDE (23) and on $\mathbb{R}$ by the propagator (32), so that in fact

$$
\begin{aligned}
G\left(y, \tau ; y_{0}, q\right)= & \int_{-\infty}^{\infty} \mathrm{d} \tilde{y} s\left(\tilde{y}, y_{0}\right) \Phi_{0}(y-\tilde{y}, \tau ; q) \\
& +\sum_{n=-\infty}^{\infty} e^{q n}\left(\Phi_{0}\left(y-y_{0}+2 n, \tau ; q\right)+e^{q y_{0}} \Phi_{0}\left(y+y_{0}+2 n, \tau ; q\right)\right) .
\end{aligned}
$$

Taking the limit $\tau \rightarrow 0$ for the entire $G\left(y, \tau ; y_{0}, q\right)$, (31) reveals

$$
\lim _{\tau \rightarrow 0} G\left(y, \tau ; y_{0}, q\right)=s\left(y, y_{0}\right)+e^{-\frac{1}{2} q\left(y-y_{0}\right)} \sum_{n=-\infty}^{\infty}\left(\delta\left(y-y_{0}+2 n\right)+\delta\left(y+y_{0}+2 n\right)\right)
$$

which means that $s\left(y, y_{0}\right)=0$ for $y, y_{0} \in[0,1]$, since $\lim _{\tau \rightarrow 0} G\left(y, \tau ; y_{0}, q\right)=\delta\left(y-y_{0}\right)$ on that interval, which implies that

$$
\sum_{n=-\infty}^{\infty} \frac{1}{k_{n}^{2}+(1 / 4) q^{2}}\left(k_{n} \sin \left(k_{n} z\right)-\frac{1}{2} q \cos \left(k_{n} z\right)\right)=-\frac{2}{e^{q}-1} e^{\frac{1}{2} q z}
$$

for any $z \in[0,2]$. Clearly, the left hand side is periodic in $z$ with period 2, which means that it is in fact the periodic repetition of the right hand side, i.e.

$$
\sum_{n=-\infty}^{\infty} \frac{1}{k_{n}^{2}+(1 / 4) q^{2}}\left(k_{n} \sin \left(k_{n} z\right)-\frac{1}{2} q \cos \left(k_{n} z\right)\right)=-\sum_{n=-\infty}^{\infty} \rho(z+2 n ; q)
$$

with

$$
\rho(z ; q)=\frac{2}{e^{q}-1} e^{\frac{1}{2} q z} I_{[0,2]}(z)
$$


and the indicator function

$$
I_{\Omega}(z)=\left\{\begin{array}{ll}
1 & \text { for } z \in \Omega \\
0 & \text { otherwise }
\end{array} .\right.
$$

On this basis, the source can be rewritten as

$$
s\left(y, y_{0}\right)=\frac{q e^{q y_{0}}}{e^{q}-1}\left(1-\sum_{n=-\infty}^{\infty} e^{q n} I_{0,2}\left(y+y_{0}+2 n\right)\right)
$$

and therefore

$$
\begin{aligned}
G\left(y, \tau ; y_{0}, q\right)= & \int_{0}^{2} d \tilde{y} \frac{q e^{q y_{0}}}{e^{q}-1} \sum_{n=-\infty}^{\infty}\left(1-e^{q n}\right) \Phi_{0}\left(y+y_{0}+2 n-\tilde{y}, \tau ; q\right) \\
& +\sum_{n=-\infty}^{\infty} e^{q n}\left(\Phi_{0}\left(y-y_{0}+2 n, \tau ; q\right)+e^{q y_{0}} \Phi_{0}\left(y+y_{0}+2 n, \tau ; q\right)\right) .
\end{aligned}
$$

Problems of convergence have not been addressed here in a detail, which could affect some of the manipulations done above, in particular when the order of integration and summation is changed. Yet, because of the free propagator $\Phi_{0}$ effectively posing an exponential cutoff on the sums as well as the integrals, all convergence issues turn out to be harmless.

To verify that the propagator (43) indeed solves the PDE (23) is a matter of a straight forward calculation. Similarly, the initial condition $\lim _{\tau \rightarrow 0} G\left(y, \tau ; y_{0}, q\right)=$ $\delta\left(y-y_{0}\right)$ for $y, y_{p} \in[0,1]$ can be identified by mere inspection. The only slight difficulty are the boundary conditions $\left.\partial_{y}\right|_{y=0,1} G\left(y, \tau ; y_{0}, q\right)=0$, which are most easily verified by writing the gradient as

$$
\begin{aligned}
\partial_{y} G\left(y, \tau ; y_{0}, q\right) & =e^{-\frac{1}{2} q\left(y-y_{0}\right)-\frac{1}{4} q^{2} \tau} \frac{1}{\sqrt{4 \pi \tau}}\left\{\sum_{n=-\infty}^{\infty} \frac{y_{0}-2 n-q \tau}{2 \tau}\left(e^{-\frac{\left(y-y_{0}+2 n\right)^{2}}{4 \tau}}-e^{-\frac{\left(-y-y_{0}+2 n\right)^{2}}{4 \tau}}\right)\right. \\
& \left.-\frac{y}{2 \tau}\left(e^{-\frac{\left(y-y_{0}+2 n\right)^{2}}{4 \tau}}+e^{-\frac{\left(-y-y_{0}+2 n\right)^{2}}{4 \tau}}\right)\right\}
\end{aligned}
$$

\subsection{Calculation of the roughness}

The propagator (43) can be used in the formal solution (22), which provides the basis for calculating the roughness via (3) and (19),

$$
w^{2}(L, t)=\frac{L \Gamma^{2}}{D} \int_{0}^{1} \mathrm{~d} y_{1} \int_{0}^{1} \mathrm{~d} y_{2}\left[\delta\left(y_{1}-y_{2}\right)-1\right] \varphi\left(y_{1}, \tau\right) \varphi\left(y_{2}, \tau\right)
$$

where the ensemble average enters only through (20).

The integral on the right is the dimensionless roughness,

$$
\int_{0}^{1} \mathrm{~d} y_{1} \int_{0}^{1} \mathrm{~d} y_{2}\left[\delta\left(y_{1}-y_{2}\right)-1\right] \varphi\left(y_{1}, \tau\right) \varphi\left(y_{2}, \tau\right)
$$

which depends on only two dimensionless parameters, $\tau=D t / L^{2}$ and $q=v L / D$. However, as discussed in the following, the parameterisation

$$
\tilde{w}^{2}(q, u)=q \int_{0}^{1} \mathrm{~d} y_{1} \int_{0}^{1} \mathrm{~d} y_{2}\left[\delta\left(y_{1}-y_{2}\right)-1\right] \varphi\left(y_{1}, \tau\right) \varphi\left(y_{2}, \tau\right)
$$


with $u=t v^{2} / D=q^{2} \tau$ is advantageous, so that $w^{2}(L, t)=\left(\Gamma^{2} / v\right) \tilde{w}^{2}(q, u)$, which means that all scaling of the roughness can be read off the scaling of $\tilde{w}^{2}$ without any further prefactor such as $L$ in (45).

The limit $t \rightarrow \infty$ in (45) translates simply to $\tau \rightarrow \infty$ in (46) and the scaling of this limit in $L$ is observed through the scaling in $q$, which is unaffected by the limit $\tau \rightarrow \infty$. So, in order to determine the roughness exponent $\alpha$, the parameterisation of (46) in $q, \tau$ is suitable. However, taking the thermodynamic limit $L \rightarrow \infty$ means $\tau \rightarrow 0$ and $q \rightarrow \infty$ simultaneously and not independently, since $q^{2} \tau=u$ remains constant.

Using $\tilde{w}^{2}\left(q=v L / D, u=t v^{2} / D\right)$ as the parameterisation of the roughness means that the two limits $L \rightarrow \infty$ and $t \rightarrow \infty$ affect each only one dimensionless parameter but not the other. The limit $t \rightarrow \infty$ means $u \rightarrow \infty$ and the scaling in large $L$ is observed through arbitrarily large but fixed $q$. The limit $L \rightarrow \infty$ means $q \rightarrow \infty$ and the scaling in large $t$ is observed through the scaling in large but fixed $u$. The two different asymptotes will be determined by considering large $q$ in both cases and $u / q$ diverging or $u / q$ vanishing.

Using the parameter $u$ in favour of $\tau$ needs to be implemented in the propagator as well, (43), using $\tau=u / q^{2}$. It is also useful to replace the integral over $\tau$ in the formal solution (22) correspondingly by an integral over $u$ so that

$$
\begin{aligned}
& \frac{v}{\Gamma^{2}} w^{2}(L, t)=\tilde{w}^{2}(q, u)=q^{-1} \int_{0}^{1} \mathrm{~d} y_{1} \int_{0}^{1} \mathrm{~d} y_{2}\left[\delta\left(y_{1}-y_{2}\right)-1\right] \\
& \times \int_{0}^{1} \mathrm{~d} y^{\prime} \int_{0}^{u} \mathrm{~d} u^{\prime} \tilde{G}\left(y_{1}, u^{\prime} ; y^{\prime}, q\right) \tilde{G}\left(y_{2}, u^{\prime} ; y^{\prime}, q\right)
\end{aligned}
$$

where $\tilde{G}$ is the re-parameterised propagator with all parameters explicitly appearing as arguments.

The bulk of the work is performing the integration (48). This can be done more conveniently by splitting the propagator up into four terms,

$$
\tilde{G}\left(y_{i}, u^{\prime} ; y^{\prime}, q\right)=\mathrm{A}_{i}-\mathrm{B}_{i}+\mathrm{C}_{i}+\mathrm{D}_{i},
$$

where

$$
\begin{aligned}
\mathrm{A}_{i} & \equiv \frac{q e^{q y^{\prime}}}{e^{q}-1} \\
\mathrm{~B}_{i} & \equiv \frac{q e^{q y^{\prime}}}{e^{q}-1} \sum_{n_{i}=-\infty}^{\infty} e^{q n_{i}} \int_{0}^{2} \mathrm{~d} \tilde{y}_{i} \tilde{\Phi}_{0}\left(y_{i}+y^{\prime}+2 n_{i}-\tilde{y}_{i}, u ; q\right) \\
\mathrm{C}_{i} & \equiv \sum_{n_{i}=-\infty}^{\infty} e^{q n_{i}} \tilde{\Phi}_{0}\left(y_{i}-y^{\prime}+2 n_{i}, u ; q\right) \\
\mathrm{D}_{i} & \equiv \sum_{n_{i}=-\infty}^{\infty} e^{q\left(n_{i}+y^{\prime}\right)} \tilde{\Phi}_{0}\left(y_{i}+y^{\prime}+2 n_{i}, u ; q\right)
\end{aligned}
$$

with

$$
\tilde{\Phi}_{0}(y, u ; q)=\frac{q}{\sqrt{4 \pi u}} e^{-\frac{(q y+u)^{2}}{4 u}} .
$$




\subsection{Integration and analysis}

Because of the prefactor $\left[\delta\left(y_{1}-y_{2}\right)-1\right]$, for each of the ten distinct terms generated by using (50) in (48), effectively two different integrations have to be performed, namely one over $y_{1}$ and $y_{2}$ distinct, and one over $y_{1}=y_{2}$. In addition, an integral over $y^{\prime}$ and $u^{\prime}$ needs to be performed, as well as over $\tilde{y}$ for term $\mathrm{B}_{i}$. In all cases, a saddle point approximation (SPA) is used, specifically

$$
\frac{q}{\sqrt{4 \pi \tilde{u}}} \int_{\mathbb{A}} \mathrm{d} y e^{-\frac{\left(q\left(y-y_{0}\right)+\tilde{u}\right)^{2}}{4 \tilde{u}}}=I_{\mathbb{A}}\left(y_{0}-\frac{\tilde{u}}{q}\right) \quad \text { for } \quad \tilde{u} / q^{2} \ll 1,
$$

where $I_{\mathbb{A}}(y)$ is again an indicator function, (41). It is worth stressing that there are no further algebraic terms, i.e. all other contributions from the integral vanish exponentially in large $q^{2} / \tilde{u}$.

In most cases, further integrals over variables contained in the argument of the indicator function are to be performed. If the intersection of the integration range of the other variables and the set $\mathbb{A}$ above has finite measure, the result is straight forward to calculate. If, however, the intersection contains a single point, then the SPA has to be considered to higher order. In general, for each "marginal variable" the resulting integral acquires an additional factor $\sqrt{u} / q$. For example, to leading order $\tilde{u} / q^{2}$,

$$
\int_{0}^{1} \mathrm{~d} y_{1} \int_{0}^{1} \mathrm{~d} y_{2} e^{-\frac{\left(q\left(y_{1}-y_{2}-1 / 3\right)\right)^{2}}{4 u}} \approx \frac{\sqrt{4 \pi u}}{q} \int_{0}^{1} \mathrm{~d} y_{2} I_{(0,1)}\left(y_{1}+1 / 3\right)=\frac{4 \sqrt{\pi u}}{3 q}
$$

using (52) in a non-marginal case (maximum of the exponential at $y_{1}-y_{2}=1 / 3 \in$ $(-1,1))$, whereas replacing $1 / 3$ by 1 produces

$$
\int_{0}^{1} \mathrm{~d} y_{1} \int_{0}^{1} \mathrm{~d} y_{2} e^{-\frac{\left(q\left(y_{1}-y_{2}-1\right)\right)^{2}}{4 u}} \approx \frac{2 u}{q^{2}}
$$

rather than $\frac{\sqrt{4 \pi u}}{q} \int_{0}^{1} \mathrm{~d} y_{2} I_{(0,1)}\left(y_{1}+1\right)=0$. Integrals like that result in subleading terms, whose amplitude is not normally calculated in the following. Instead, only the power of $q$ is noted and by comparison to other terms it is verified that it is safe to ignore it. The same result is recovered by power counting in the four terms (50), where each integral gives rise to a leading order $q^{-1}$ and thus each of the four terms has the same algebraic dependence on $q$.

Further details of the calculation are exemplified in the appendix. Combining all contributions gives

$$
\begin{aligned}
w_{\text {physical }}^{2}= & \frac{\Gamma^{2}}{v} q^{-1} \int_{0}^{1} \mathrm{~d} y_{1} \int_{0}^{1} \mathrm{~d} y_{2}\left[\delta\left(y_{1}-y_{2}\right)-1\right] \\
& \times\left(\mathrm{A}_{1}-\mathrm{B}_{1}+\mathrm{C}_{1}+\mathrm{D}_{1}\right)\left(\mathrm{A}_{2}-\mathrm{B}_{2}+\mathrm{C}_{2}+\mathrm{D}_{2}\right)
\end{aligned}
$$

For $u$ small, looking at the limit $q \rightarrow \infty$, to leading order:

$$
\lim _{q \rightarrow \infty} w_{\text {physical }}^{2}=\frac{\Gamma^{2}}{v} \sqrt{\frac{u}{2 \pi}}
$$

For $q$ large and considering the limit $u \rightarrow \infty$, to leading order:

$$
\lim _{u \rightarrow \infty} w_{\text {physical }}^{2}=\frac{\Gamma^{2}}{v} \frac{2}{3 \sqrt{2 \pi}} \sqrt{q}
$$




\section{Discussion and conclusion}

Upon replacing $u$ and $q$ by their definitions, $u=t v^{2} / D$ and $q=v L / D$, the two asymptotes for the roughness derived in Equation (56) and (55) are

$$
w^{2}(t, L)=\Gamma^{2} \times\left\{\begin{aligned}
\sqrt{\frac{t}{2 \pi D}} & \text { for } L \rightarrow \infty \\
\frac{2}{3} \sqrt{\frac{L}{2 \pi D v}} & \text { for } t \rightarrow \infty
\end{aligned}\right.
$$

which is, to leading order, identical to the result in [5] for Dirichlet boundary conditions. The exponents as defined in (6),

$$
\alpha=\frac{1}{4}, \quad \beta=\frac{1}{4}, \quad z=1 \quad(\text { EWd with Neumann BC) }
$$

therefore reproduce (16). On the one hand, this is a surprising result, because after realising that the usual dimensional arguments do no longer apply, any exponents are mathematically possible. The fact that Neumann boundary conditions reproduce the anomalous results of the Dirichlet case (contrasting those for periodic boundary conditions) even down to the amplitudes therefore point to some universal mechanism at work in both equations. Physically, on the other hand, arguments very similar to those discussed in [5] apply: The drift with velocity $v$ effectively constantly re-initialises the interface with vanishing slope from one side to the other, while constantly under the influence of the external noise. This mechanism erases practically all features that develop over times exceeding $L / v$ and thus in saturation, $t \rightarrow \infty$ reduces the roughness to about the value after time $L / v$. In fact, the stationary roughness is $2 / 3$ of what is extrapolated from initial roughening:

$$
\lim _{t \rightarrow \infty} w^{2}(t, L)=\frac{2}{3} \lim _{L^{\prime} \rightarrow \infty} w^{2}\left(L / v, L^{\prime}\right)
$$

In conclusion we have shown that the Edwards-Wilkinson equation with drift and Neumann boundary conditions produces anomalous scaling different from what is expected from naive dimensional analysis and easily derived for periodic boundary conditions. The scaling and the amplitudes of the Dirichlet case are reproduced, consistent with a simple physical scenario of an interface that is constantly re-initialised. Neumann boundary conditions are physically more relevant than Dirichlet ones, yet far more difficult to handle analytically.

\section{Acknowledgments}

GP would like to thank Vikram Pandya for bringing his attention to this problem. We thank D. E. Khmelnitskii for pointing out a mistake in an earlier version of this manuscript. 
The Edwards-Wilkinson equation with drift and Neumann boundary conditions

\section{Appendix A. Integral (48) term by term}

In this appendix some details of the integral (48) are shown, considering the propagator term by term as defined in (49) and (50).

Appendix A.1. $\mathrm{A}_{1} \mathrm{X}_{2}$ Term

Since there is no $y_{1}$ or $y_{2}$ dependence in $A_{1}$, (50a):

$$
\int_{0}^{1} \mathrm{~d} y_{1} \underbrace{\int_{0}^{1} \mathrm{~d} y_{2}\left[\delta\left(y_{1}-y_{2}\right)-1\right]}_{\equiv 0} \int_{0}^{1} \mathrm{~d} y^{\prime} \int_{0}^{u} \mathrm{~d} u^{\prime} \mathrm{A}_{1} \mathrm{X}_{2}=0
$$

for $\mathrm{X}$ being any of the $\mathrm{A}, \mathrm{B}, \mathrm{C}$ or $\mathrm{D}$ terms.

Appendix A.2. $\mathrm{B}_{1} \mathrm{X}_{i}$ Term

Since all $\mathrm{B}_{1} \mathrm{X}_{i}$ integrals follow similar arguments, we exemplify the procedure on $\mathrm{B}_{1} \mathrm{C}_{2}$. The integrals to be calculated (to leading order) are

$$
\begin{aligned}
& w_{\mathrm{BC}}^{2}=\frac{\Gamma^{2}}{v} q^{-1} \int_{0}^{1} \mathrm{~d} y_{1} \int_{0}^{1} \mathrm{~d} y_{2}\left[\delta\left(y_{1}-y_{2}\right)-1\right] \int_{0}^{1} \mathrm{~d} y^{\prime} \int_{0}^{u} \mathrm{~d} u^{\prime} \\
& \times \frac{q e^{q y^{\prime}}}{e^{q}-1} \sum_{n_{1}=-\infty}^{\infty} e^{q n_{1}} \int_{0}^{2} \mathrm{~d} \tilde{y}_{1} \frac{q}{\sqrt{4 \pi u}} e^{-\frac{\left(q\left(y_{1}+y^{\prime}+2 n_{1}-\tilde{y}_{1}\right)+u\right)^{2}}{4 u}} \sum_{n_{2}=-\infty}^{\infty} e^{q n_{2}} \frac{q}{\sqrt{4 \pi u}} e^{-\frac{\left(q\left(y_{2}-y^{\prime}+2 n_{2}\right)+u\right)^{2}}{4 u}} .
\end{aligned}
$$

We consider the total exponential of $B_{1} C_{2}$ in the form

$$
\frac{1}{e^{q}-1} \exp \left(q\left(n_{1}+n_{2}\right)-f\left(y^{\prime}, \tilde{y}\right)\right)
$$

where

$$
f\left(y^{\prime}, \tilde{y}\right)=-q y^{\prime}+\frac{q^{2}}{4 u^{\prime}}\left(y_{1}+y^{\prime}+2 n_{1}-\tilde{y}+\frac{u^{\prime}}{q}\right)^{2}+\frac{q^{2}}{4 u^{\prime}}\left(y_{2}-y^{\prime}+2 n_{2}+\frac{u^{\prime}}{q}\right)^{2} .
$$

A contribution in the form (A.2) vanishes for large $q$ unless

$$
n_{1}+n_{2}-\frac{f\left(y^{\prime}, \tilde{y}\right)}{q} \geq 1 .
$$

Applying the SPA to the integral over $y^{\prime}$, means to evaluate the integral at $y^{\prime}$ that minimises $f$ :

$$
y_{0}^{\prime}=\frac{1}{2}\left(y_{2}-y_{1}\right)+\left(n_{2}-n_{1}\right)+\frac{1}{2} \tilde{y}+\frac{u^{\prime}}{q},
$$

where $f$ now becomes:

$$
f\left(y_{0}^{\prime}, \tilde{y}\right)=\frac{q^{2}}{2 u^{\prime}}\left(\frac{1}{2}\left(y_{1}+y_{2}\right)+\left(n_{1}+n_{2}\right)-\frac{\tilde{y}}{2}\right)^{2}+q\left(y_{1}+2 n_{1}-\tilde{y}\right) .
$$

Applying the SPA again to the integral over $\tilde{y}$ gives, with $f\left(y_{0}^{\prime}, \tilde{y}\right)$ above,

$$
\tilde{y}_{0}=y_{1}+y_{2}+2\left(n_{1}+n_{2}\right)+\frac{4 u^{\prime}}{q},
$$


where now

$$
f\left(y_{0}^{\prime}, \tilde{y}_{0}\right)=-q\left(y_{2}+2 n_{2}\right)-2 u^{\prime} .
$$

Determining the asymptotic behaviour is greatly facilitated by writing $\frac{u^{\prime}}{q}=\sigma+m \geq 0$, with $\sigma \in[0,1)$ and $m \in \mathbb{N}^{0}$, so that $\left\lfloor u^{\prime} / q\right\rfloor=m$. Using the final result of $f$, (A.8), the inequality in (A.4) becomes:

$$
n_{1}+n_{2}-\frac{f}{q}=y_{2}+2 \sigma+n_{1}+3 n_{2}+2 m \geq 1 \text {. }
$$

As $y_{2} \in[0,1]$ and $\sigma \in[0,1)$, so $y_{2}+2 \sigma \in[0,3)$, the contribution (A.2) vanishes for large $q$ unless

$$
n_{1}+3 n_{2}+2 m>-2 .
$$

Since $n_{1}, n_{2}$ and $m$ are integers, we have the inequality

$$
n_{1}+3 n_{2}+2 m \geq-1 \text {. }
$$

Both SPAs produce an indicator function as well. Writing $\frac{u^{\prime}}{q}$ in terms of $\sigma$ and $m$ gives

$$
\begin{aligned}
& \mathrm{I}_{(0,1)}\left(y_{0}^{\prime}\right)=\mathrm{I}_{(0,1)}\left[\left(y_{2}-y_{1}\right) / 2+\left(n_{2}-n_{1}\right)+\tilde{y} / 2+\sigma+m\right] \\
& \mathrm{I}_{(0,2)}\left(\tilde{y}_{0}\right)=\mathrm{I}_{(0,2)}\left[y_{1}+y_{2}+2\left(n_{1}+n_{2}\right)+4 \sigma+4 m\right] .
\end{aligned}
$$

From the indicator function of $y_{0}^{\prime}$ above, (A.11), we have

$$
0<\frac{1}{2}\left(y_{2}-y_{1}\right)+\left(n_{2}-n_{1}\right)+\frac{1}{2} \tilde{y}+\sigma+m<1
$$

as a condition for (A.2) to contribute. As $y_{1}, y_{2} \in[0,1), \tilde{y} \in[0,2)$ and $\sigma \in[0,1)$, this implies that $\frac{1}{2}\left(y_{2}-y_{1}\right)+\frac{1}{2} \tilde{y}+\sigma \in\left[-\frac{1}{2}, \frac{5}{2}\right)$. By subtracting this range, (A.13) becomes $-\frac{5}{2}<n_{2}-n_{1}+m<\frac{3}{2}$, which gives

$$
-2 \leq n_{2}-n_{1}+m \leq 1
$$

as $n_{1}, n_{2}$ and $m$ are integers. Similarly, for (A.12) to be non-zero, we have the condition

$$
-2 \leq\left(n_{1}+n_{2}\right)+2 m \leq 0 .
$$

$\mathrm{B}_{1} \mathrm{C}_{2}$ vanishes if either one of these two ranges, (A.14) and (A.15), is incompatible with all possible values of $n_{1}, n_{2}$ and $m$. By adding (A.10) and (A.14) we find $-3 \leq 4 n_{2}+3 m$ and as $m \in \mathbb{N}^{0}$ it follows $n_{2} \geq 0$. Adding (A.14) and (A.15), we obtain $-4 \leq 2 n_{2}+3 m \leq 1$ and with $m \geq 0$ it now follows $n_{2}=m=0$ to prevent either indicator function from vanishing. Therefore, the two indicator functions become

$$
\begin{aligned}
& \mathrm{I}_{(0,1)}\left(y_{0}^{\prime}\right)=\mathrm{I}_{(0,1)}\left[y_{2}+3 \sigma\right] \\
& \mathrm{I}_{(0,2)}\left(\tilde{y}_{0}\right)=\mathrm{I}_{(0,2)}\left[y_{1}+y_{2}+2 n_{1}+4 \sigma\right] .
\end{aligned}
$$

The next variable to determine is $n_{1}$. With $n_{2}=m=0$ Equation (A.9) reads:

$$
y_{2}+2 \sigma+n_{1}>1
$$

Multiplying (A.18) by 2 and rearranging, we have the condition $y_{2}+4 \sigma+2 n_{1}>2-y_{2}>1$, as $y_{2} \in[0,1)$, and thus

$$
y_{2}+3 \sigma>1-\sigma-2 n_{1}
$$


Using $n_{2}=m=0$ in (A.10) and (A.15) gives the range of $n_{1}$ as $-1 \leq n_{1} \leq 0$, whereas (A.16) indicates

$$
1>y_{2}+3 \sigma>0 \text {. }
$$

If $n_{1}=-1$, then (A.19) becomes $y_{2}+3 \sigma>3-\sigma$. Since $\sigma \in[0,1)$, this contradicts (A.20). Therefore, we conclude that $n_{1}=0$. However, if we use the fact that $n_{1}=n_{2}=m=0$ in (A.9) we get $y_{2}+2 \sigma>1$. By comparing to (A.20), we have $\sigma<0$ which contradicts the fact that $\sigma \in[0,1)$. As the conditions cannot be fulfilled simultaneously, this implies that the integrals vanish exponentially in large $q$.

Considering in addition any marginal cases will produce terms of lower algebraic order in $q$. For the present integral, the marginal cases are $n_{1}=n_{2}=m=0$ with $y_{2}=0$ and $\sigma=0$ (double marginal), as well as $n_{1}=1, n_{2}=m=0$ with $y_{1}=y_{2}=0$ and $\sigma=0$ (triple marginal). Power counting in the initial integral (A.1) thus gives overall contributions to $w_{\mathrm{BC}}^{2}$ of order $\mathcal{O}\left(q^{-2}\right)$ and $\mathcal{O}\left(q^{-3}\right)$ respectively. Extra care must be taken when considering the integral with upper bound $u$, as $u \rightarrow \infty$ might be taken before $q \rightarrow \infty$, in which case the integral over $u^{\prime}$ might give rise to a term of order $q$ itself (see, for example, (A.26) versus (A.27) ). In the present case this does not apply, because $u^{\prime}=q(m+\sigma)$ and both $m$ as well as $\sigma$ are fixed by the SPA.

By similar arguments, the terms $\mathrm{B}_{1} \mathrm{~B}_{2}$ and $\mathrm{B}_{1} \mathrm{D}_{2}$ vanish.

Appendix A.3. $\mathrm{C}_{1} \mathrm{X}_{2}$ and $\mathrm{D}_{1} \mathrm{D}_{2}$ Term

Calculations for the terms $C_{1} X_{2}$ and $D_{1} D_{2}$ are very similar and we therefore exemplify the procedure for $\mathrm{D}_{1} \mathrm{D}_{2}$ only.

We write contribution by $\mathrm{D}_{1} \mathrm{D}_{2}$ as

$$
\begin{aligned}
& w_{\mathrm{DD}}^{2}=\frac{\Gamma^{2}}{v} q^{-1} \int_{0}^{1} \mathrm{~d} y_{1} \int_{0}^{1} \mathrm{~d} y_{2}\left[\delta\left(y_{1}-y_{2}\right)-1\right] \int_{0}^{1} \mathrm{~d} y^{\prime} \int_{0}^{u} \mathrm{~d} u^{\prime} \\
& \times \sum_{n_{1}=-\infty}^{\infty} e^{q n_{1}} \frac{q}{\sqrt{4 \pi u^{\prime}}} e^{\frac{-\left[q\left(y_{1}+y^{\prime}+2 n_{1}\right)+u^{\prime}\right]^{2}}{4 u^{\prime}}} e^{q y^{\prime}} \sum_{n_{2}=-\infty}^{\infty} e^{q n_{2}} \frac{q}{\sqrt{4 \pi u^{\prime}}} e^{\frac{-\left[q\left(y_{2}+y^{\prime}+2 n_{2}\right)+u^{\prime}\right]^{2}}{4 u^{\prime}}} e^{q y^{\prime}}
\end{aligned}
$$

We consider the total exponent of $\mathrm{D}_{1} \mathrm{D}_{2}$ in the form

$$
\exp \left(q\left(n_{1}+n_{2}\right)-f\right)
$$

We first apply the SPA to the integral over $y^{\prime}$, and obtain the minimum $y_{0}^{\prime}$, so that

$f\left(y_{0}^{\prime}\right)=\frac{q^{2}}{2 u^{\prime}}\left[\frac{1}{2}\left(y_{1}-y_{2}\right)+\left(n_{1}-n_{2}\right)\right]^{2}+2 q\left[\frac{1}{2}\left(y_{1}+y_{2}\right)+\left(n_{1}+n_{2}\right)\right]$.

Again, the analysis of the asymptotic behaviour is greatly facilitated by writing $\frac{u^{\prime}}{q}=\sigma+m$, with $\sigma \in[0,1)$ and $m \in \mathbb{N}^{0}$, so that the indicator function for $y_{0}^{\prime}$ is

$$
\mathrm{I}_{(0,1)}\left(y_{0}^{\prime}\right)=\mathrm{I}_{(0,1)}\left(\sigma-\frac{1}{2}\left(y_{1}+y_{2}\right)+m-\left(n_{1}+n_{2}\right)\right) .
$$

Since $\sigma-\frac{1}{2}\left(y_{1}+y_{2}\right) \in[-1,1)$, the remainder of the argument $m-\left(n_{1}+n_{2}\right)$ must be greater than -1 or less than 2 for this term to contribute. Therefore we will only need 
to consider only $n_{1}+n_{2}=m$ and $n_{1}+n_{2}=m-1$, incorporated below by means of Kronecker $\delta$-functions. The total contribution then becomes:

$$
\begin{aligned}
w_{\mathrm{DD}}^{2}=\frac{\Gamma^{2}}{v} q^{-1} & \int_{0}^{1} \mathrm{~d} y_{1} \int_{0}^{1} \mathrm{~d} y_{2}\left[\delta\left(y_{1}-y_{2}\right)-1\right] \\
& \times \int_{0}^{u} \mathrm{~d} u^{\prime} \sum_{n_{1}, n_{2}} \frac{q}{\sqrt{8 \pi u^{\prime}}} \exp \left\{-\frac{q^{2}}{2 u^{\prime}}\left[\frac{1}{2}\left(y_{1}-y_{2}\right)+\left(n_{1}-n_{2}\right)\right]^{2}\right. \\
& \left.-2 q\left[\frac{1}{2}\left(y_{1}+y_{2}\right)+\left(n_{1}+n_{2}\right)\right]\right\}\left\{e^{-q m} \mathrm{I}_{(0,1)}\left[-\frac{1}{2}\left(y_{1}+y_{2}\right)+\sigma\right] \delta_{m, n_{1}+n_{2}}\right. \\
& \left.+e^{-q(1-m)} \mathrm{I}_{(-1,0)}\left[-\frac{1}{2}\left(y_{1}+y_{2}\right)+\sigma\right] \delta_{m-1, n_{1}+n_{2}}\right\} .
\end{aligned}
$$

At this stage, it is sensible to consider the $\delta$-term and 1-term in $\left[\delta\left(y_{1}-y_{2}\right)-1\right]$ separately. For $y_{1}=y_{2}=y$, i.e. the contribution of the $\delta$-term, the integral to consider is

$$
\begin{gathered}
\left(\mathrm{D}_{1} \mathrm{D}_{2}\right)_{\delta}=\int_{0}^{1} \mathrm{~d} y \int_{0}^{u} \mathrm{~d} u^{\prime} \sum_{n_{1}, n_{2}} \frac{q}{\sqrt{8 \pi u^{\prime}}} \exp \left(-\frac{q^{2}}{2 u^{\prime}}\left(n_{1}-n_{2}\right)^{2}-2 q\left(y+\left(n_{1}+n_{2}\right)\right)\right) \\
\times\left[e^{-q m} \mathrm{I}_{(0,1)}[\sigma-y] \delta_{m, n_{1}+n_{2}}+e^{-q(1-m)} \mathrm{I}_{(-1,0)}[\sigma-y] \delta_{m-1, n_{1}+n_{2}}\right] .
\end{gathered}
$$

using $\mathrm{I}_{(0,1)}(y+1)=\mathrm{I}_{(-1,0)}(y)$. This term can in turn be split into three parts, each accounting for one of the Kronecker $\delta$-functions and the possible values of $m$. To prevent exponential suppression, $m=0$ is required for the prefactor $e^{-q m}$ and $m=0,1$ for the prefactor $e^{-q(1-m)}$, which gives rise to three terms, $\left(\mathrm{D}_{1} \mathrm{D}_{2}\right)_{\delta}=$ $\left(\mathrm{D}_{1} \mathrm{D}_{2}\right)_{\delta_{1}}+\left(\mathrm{D}_{1} \mathrm{D}_{2}\right)_{\delta_{2}}+\left(\mathrm{D}_{1} \mathrm{D}_{2}\right)_{\delta_{3}}$.

In the first term $\left(\mathrm{D}_{1} \mathrm{D}_{2}\right)_{\delta_{1}}$ we have $m=0$ and $n_{1}=-n_{2}$ from the Kronecker $\delta$. Ensuring the indicator function is non-zero, we change the integration limits of $y$ and using $\delta_{0, n_{1}+n_{2}}$ we write $n_{1}=-n_{2}=n$,

$$
\begin{aligned}
\left(\mathrm{D}_{1} \mathrm{D}_{2}\right)_{\delta_{1}}=\int_{0}^{\sigma} \mathrm{d} y \int_{0}^{u} \mathrm{~d} u^{\prime} \sum_{n} \frac{q}{\sqrt{8 \pi u^{\prime}}} \exp \left(-\frac{q^{2}}{u^{\prime}}\left(2 n^{2}\right)-2 q y\right) \\
=\int_{0}^{u} \mathrm{~d} u^{\prime} \sum_{n} \frac{1}{2 q} \exp \left(-\frac{q^{2}}{u^{\prime}}\left(2 n^{2}\right)\right) \frac{q}{\sqrt{8 \pi u^{\prime}}}\left[1-e^{-2 q \sigma}\right] .
\end{aligned}
$$

In the limit of large $q$, the first exponential will asymptotically vanish unless $n=0$. Since $\left\lfloor u^{\prime} / q\right\rfloor=m=0$, we change the upper integration limit of $u^{\prime}$ to $\min (q, u)$, and apply $n=0$ :

$$
\begin{aligned}
\left(\mathrm{D}_{1} \mathrm{D}_{2}\right)_{\delta_{1}} & =\int_{0}^{\min \left(1, \frac{u}{q}\right)} q \mathrm{~d} \sigma \sum_{n} \frac{1}{2 q} \exp \left(-\frac{q^{2}}{u^{\prime}}\left(2 n^{2}\right)\right) \frac{q}{\sqrt{8 \pi q}} \frac{1}{\sqrt{\sigma}}\left[1-e^{-2 q \sigma}\right] \\
& =\frac{1}{4} \sqrt{\frac{q}{2 \pi}} \int_{0}^{\min \left(1, \frac{u}{q}\right)} \mathrm{d} \sigma \frac{1}{\sqrt{\sigma}}\left[1-e^{-2 q \sigma}\right] \\
& =\frac{1}{4} \sqrt{\frac{q}{2 \pi}}\left[2 \min \left(1, \frac{u}{q}\right)-\frac{1}{\sqrt{2 q}} \Gamma\left(\frac{1}{2}, 2 q \min \left(1, \frac{u}{q}\right)\right)\right]
\end{aligned}
$$

using $u^{\prime}=\sigma q$ and

$$
\int_{0}^{y} \mathrm{~d} x x^{\mu} e^{-x}=\Gamma(\mu+1, y),
$$

which converges to $\sqrt{\pi}$ for $\mu=1 / 2$ in the limit of large $y$. 
The Edwards-Wilkinson equation with drift and Neumann boundary conditions

Similar arguments can be used for $\left(\mathrm{D}_{1} \mathrm{D}_{2}\right)_{\delta_{2}}$. In that case, the support of the indicator function changes from $[0,1]$ to $[-1,0]$ since $m=1$ and therefore the integral changes to:

$$
\begin{aligned}
\left(\mathrm{D}_{1} \mathrm{D}_{2}\right)_{\delta_{2}} & =\int_{\sigma}^{\infty} \mathrm{d} y \int_{0}^{u} \mathrm{~d} u^{\prime} \sum_{n} \frac{q}{\sqrt{8 \pi u^{\prime}}} \exp \left(-\frac{q^{2}}{u^{\prime}}\left(2 n^{2}\right)-2 q y\right) \\
& =\frac{1}{4} \sqrt{\frac{q}{2 \pi}}\left[\frac{1}{\sqrt{2 q}} \Gamma\left(\frac{1}{2}, 2 q \min \left(1, \frac{u}{q}\right)\right)\right] .
\end{aligned}
$$

Finally, the contribution of $\left(\mathrm{D}_{1} \mathrm{D}_{2}\right)_{\delta_{3}}$, with $m=0$, is

$$
\begin{aligned}
\left(\mathrm{D}_{1} \mathrm{D}_{2}\right)_{\delta_{3}}= & \int_{0}^{1} \mathrm{~d} y \int_{0}^{u} \mathrm{~d} u^{\prime} \sum_{n_{1}, n_{2}} \frac{q}{\sqrt{8 \pi u^{\prime}}} \\
& \times \exp \left(-\frac{q^{2}}{2 u^{\prime}}\left(n_{1}-n_{2}\right)^{2}-2 q\left(y+\left(n_{1}+n_{2}\right)\right)\right) e^{-q} \mathrm{I}_{(-1,0)}(\sigma-y) .
\end{aligned}
$$

Since $m=0$, this means that $n_{1}+n_{2}=-1$. Letting $n_{1}=n$, this gives $n_{1}-n_{2}=2 n+1$ and thus

$$
\begin{aligned}
\left(\mathrm{D}_{1} \mathrm{D}_{2}\right)_{\delta_{3}}= & \int_{0}^{1} \mathrm{~d} y \int_{0}^{u} \mathrm{~d} u^{\prime} \sum_{n} \frac{q}{\sqrt{8 \pi u^{\prime}}} \exp \left(-q\left(\frac{q}{2 u^{\prime}}(2 n+1)^{2}+2 y-1\right)\right) \\
& \times e^{-q} \mathrm{I}_{(-1,0)}(\sigma-y) \ldots
\end{aligned}
$$

Each exponential in the sum vanishes for large $q$ unless

$$
\frac{q}{2 u^{\prime}}(2 n+1)^{2}+2 y-1 \leq 0
$$

Since $m=0, u^{\prime}=q \sigma<q$, and from the indicator function follows $y>\sigma$, so that

$$
0 \geq \frac{1}{2 \sigma}(2 n+1)^{2}+2 y-1 \geq \frac{1}{2 \sigma}+2 y-1>\frac{1}{2 \sigma}+2 \sigma-1
$$

which implies $-2 \sigma \geq(2 \sigma-1)^{2}$ for $\sigma \geq 0$, which is impossible. The $\left(\mathrm{D}_{1} \mathrm{D}_{2}\right)_{\delta_{3}}$ term therefore vanishes.

In summary we have

$$
\left(\mathrm{D}_{1} \mathrm{D}_{2}\right)_{\delta}=\left(\mathrm{D}_{1} \mathrm{D}_{2}\right)_{\delta_{1}}+\left(\mathrm{D}_{1} \mathrm{D}_{2}\right)_{\delta_{2}}+\left(\mathrm{D}_{1} \mathrm{D}_{2}\right)_{\delta_{3}}=\frac{1}{2} \sqrt{\frac{q}{2 \pi}}\left[\min \left(1, \frac{u}{q}\right)\right] \text {. }
$$

For $u$ small and by taking the limit $q \rightarrow \infty$, it is obvious that:

$$
\lim _{q \rightarrow \infty}\left(\mathrm{D}_{1} \mathrm{D}_{2}\right)_{\delta} \rightarrow 0
$$

Yet, taking $u \rightarrow \infty$ first gives $\min \left(1, \frac{u}{q}\right)=1$ and we find to leading order in $q$ :

$$
\lim _{u \rightarrow \infty}\left(\mathrm{D}_{1} \mathrm{D}_{2}\right)_{\delta}=\frac{\Gamma^{2}}{v} q^{-1} \frac{1}{2} \sqrt{\frac{q}{2 \pi}}=\frac{\Gamma^{2}}{v} \frac{1}{2 \sqrt{2 \pi q}}
$$

Next we consider the roughness contribution of the 1-term, i.e. $y_{1} \neq y_{2}$ :

$$
\begin{aligned}
\left(\mathrm{D}_{1} \mathrm{D}_{2}\right)_{1}=\int_{0}^{1} \mathrm{~d} y_{1} \int_{0}^{1} \mathrm{~d} y_{2} \int_{0}^{u} \mathrm{~d} u^{\prime} \sum_{n_{1}, n_{2}} \frac{q}{\sqrt{8 \pi u^{\prime}}} \exp \left(-\frac{q^{2}}{2 u^{\prime}}\left[\frac{1}{2}\left(y_{1}-y_{2}\right)+\left(n_{1}-n_{2}\right)\right]\right]^{2} \\
\left.-2 q\left(\frac{1}{2}\left(y_{1}+y_{2}\right)+\left(n_{1}+n_{2}\right)\right)\right)\left[e^{-q m} \mathrm{I}_{(0,1)}\left[-\frac{1}{2}\left(y_{1}+y_{2}\right)+\sigma\right] \delta_{m, n_{1}+n_{2}}\right. \\
\left.+e^{-q(m-1)} \mathrm{I}_{(-1,0)}\left[-\frac{1}{2}\left(y_{1}+y_{2}\right)+\sigma\right] \delta_{m-1, n_{1}+n_{2}}\right]
\end{aligned}
$$


This term, again, can be split into three parts, $\left(\mathrm{D}_{1} \mathrm{D}_{2}\right)_{1}=\left(\mathrm{D}_{1} \mathrm{D}_{2}\right)_{1_{1}}+\left(\mathrm{D}_{1} \mathrm{D}_{2}\right)_{1_{2}}+$ $\left(\mathrm{D}_{1} \mathrm{D}_{2}\right)_{1_{3}}$. Using similar as for the $\delta$-term, $\left(\mathrm{D}_{1} \mathrm{D}_{2}\right)_{1_{1}}$ contribution vanishes for large $q$ unless $m=0$, which implies $n_{1}=-n_{2}=n$. An SPA applied to the integral over $y_{1}$ gives a minimum at

$$
y_{1_{0}}=y_{2}-\frac{4 u^{\prime}}{q}-4 n
$$

so that the exponent is

$$
f\left(y_{1_{0}}\right)=q\left(2 y_{2}-\frac{2 u^{\prime}}{q}-4 n\right) .
$$

in the notation introduced above. The first term therefore gives

$$
\begin{aligned}
\left(\mathrm{D}_{1} \mathrm{D}_{2}\right)_{1_{1}}= & \int_{0}^{1} \mathrm{~d} y_{2} \sum_{n} \int_{0}^{u} \mathrm{~d} u^{\prime} \frac{q}{\sqrt{8 \pi u^{\prime}}} \sqrt{\frac{\pi}{q^{2} / 8 u^{\prime}}} \exp \left(-q\left(2 y_{2}-\frac{2 u^{\prime}}{q}-4 n\right)\right) \\
& \times \mathrm{I}_{(0,1)}\left[\frac{-1}{2}\left(y_{2}-\frac{4 u^{\prime}}{q}-4 n+y_{2}\right)+\sigma\right] \mathrm{I}_{(0,1)}\left[y_{2}-\frac{4 u^{\prime}}{q}-4 n\right] \\
& =\int_{0}^{1} \mathrm{~d} y_{2} \int_{0}^{u} \mathrm{~d} u^{\prime} \exp \left(-q\left(2 y_{2}-2 \sigma-4 n\right)\right) \\
& \times \mathrm{I}_{(0,1)}\left[-y_{2}+3 \sigma+2 n\right] \mathrm{I}_{(0,1)}\left[y_{2}-4 \sigma-4 n\right]
\end{aligned}
$$

Both indicator functions suggest $n=0$ or $n=-1$, otherwise the $\left(\mathrm{D}_{1} \mathrm{D}_{2}\right)_{1_{1}}$ term will not contribute. In the limit of large $q$, the term will be exponentially suppressed for $n=-1$ so that the only case to be considered is $n=1$. However, using $n=0$ in both indicator functions implies $\sigma<0$ for this term to contribute which contradicts $\sigma \geq 0$. We conclude that $\left(D_{1} D_{2}\right)_{1}$ does not contribute.

By observation, $\left(D_{1} D_{2}\right)_{12}$ is identical to $\left(D_{1} D_{2}\right)_{1}$ with the domain of the indicator function changing from $[0,1]$ to $[-1,0]$ as $m=1$.

$$
\begin{aligned}
\left(\mathrm{D}_{1} \mathrm{D}_{2}\right)_{1_{2}}= & \int_{0}^{1} \\
\mathrm{~d} & y_{2} \sum_{n} \int_{0}^{u} \mathrm{~d} u^{\prime} \frac{q}{\sqrt{8 \pi u^{\prime}}} \sqrt{\frac{\pi}{q^{2} / 8 u^{\prime}}} \exp \left(-q\left(2 y_{2}-\frac{2 u^{\prime}}{q}-4 n\right)\right) \\
& \times \mathrm{I}_{(-1,0)}\left[\frac{-1}{2}\left(y_{2}-\frac{4 u^{\prime}}{q}-4 n+y_{2}\right)+\sigma\right] \mathrm{I}_{(0,1)}\left[y_{2}-\frac{4 u^{\prime}}{q}-4 n\right] \\
& =\int_{0}^{1} \mathrm{~d} y_{2} \int_{0}^{u} \mathrm{~d} u^{\prime} \exp \left(-q\left(2 y_{2}-2 m-2 \sigma-4 n\right)\right) \\
& \times \mathrm{I}_{(-1,0)}\left[-y_{2}+3 \sigma+3 m+2 n\right] \mathrm{I}_{(0,1)}\left[y_{2}-4 \sigma-4 m-4 n\right] \\
& =\int_{0}^{1} \mathrm{~d} y_{2} \int_{0}^{u} \mathrm{~d} u^{\prime} \exp \left(-q\left(2 y_{2}-2-2 \sigma-4 n\right)\right) \\
& \times \mathrm{I}_{(-4,-3)}\left[-y_{2}+3 \sigma+2 n\right] \mathrm{I}_{(4,5)}\left[y_{2}-4 \sigma-4 n\right] .
\end{aligned}
$$

Both indicator functions contribute only if $n=-2$, in which case, however, the term vanishes exponentially in large $q$.

Finally, we consider the $\left(\mathrm{D}_{1} \mathrm{D}_{2}\right)_{1_{3}}$ contribution, for which $m=0$ and $n_{1}+n_{2}=-1$. By writing $n=n_{1}=-1-n_{2}$ and applying SPA to the integral over $y_{1}$ we find the minimum of the exponent at

$$
y_{1_{0}}=y_{2}-\frac{4 u^{\prime}}{q}-2(2 n+1)=y_{2}-4 \sigma-2(2 n+1)
$$


so that it becomes

$$
f\left(y_{1_{0}}\right)=q\left(2 y_{2}-\frac{2 u^{\prime}}{q}-4 n-1\right)=q\left(2 y_{2}-2 \sigma-4 n-1\right) .
$$

and thus

$$
\begin{aligned}
\left(\mathrm{D}_{1} \mathrm{D}_{2}\right)_{1_{3}}= & \int_{0}^{1} \mathrm{~d} y_{2} \sum_{n} \int_{0}^{u} \mathrm{~d} u^{\prime} \frac{q}{\sqrt{8 \pi u^{\prime}}} \sqrt{\frac{\pi}{q^{2} / 8 u^{\prime}}} \exp \left(-q\left(2 y_{2}-2 \sigma-4 n-1\right)\right) \\
& \times \mathrm{I}_{(-1,0)}\left[\frac{-1}{2}\left(y_{2}-4 \sigma-2(2 n+1)+y_{2}\right)+\sigma\right] \mathrm{I}_{(0,1)}\left[y_{2}-4 \sigma-2(2 n+1)\right] \\
& =\int_{0}^{1} \mathrm{~d} y_{2} \int_{0}^{u} \mathrm{~d} u^{\prime} \exp \left(-q\left(2 y_{2}-2 \sigma-4 n-1\right)\right) \\
& \times \mathrm{I}_{(-2,-1)}\left[-y_{2}+3 \sigma+2 n\right] \mathrm{I}_{(2,3)}\left[y_{2}-4 \sigma-4 n\right] .
\end{aligned}
$$

Similar arguments as for $\left(D_{1} D_{2}\right)_{12}$ apply. Both indicator functions contribute only if $n=-1$, where the term is exponentially suppressed. Therefore, $\left(D_{1} D_{2}\right)_{1_{3}}$ does not contribute either.

Hence in total, considering both limits we have to leading order:

$$
\lim _{q \rightarrow \infty} w_{\mathrm{DD}}^{2}=0 \quad \text { and } \quad \lim _{u \rightarrow \infty} w_{\mathrm{DD}}^{2}=\frac{\Gamma^{2}}{v} \frac{1}{2 \sqrt{2 \pi q}}
$$

Any marginal cases are necessarily subleading compared to (A.25) and thus can be safely ignored.

By similar procedures, we obtain the leading order behaviour of $w_{\mathrm{CC}}^{2}$ and $w_{\mathrm{CD}}^{2}$ as follows:

$$
\lim _{q \rightarrow \infty} w_{\mathrm{CC}}^{2}=\frac{\Gamma^{2}}{v} \sqrt{\frac{u}{2 \pi}} \text { and } \lim _{u \rightarrow \infty} w_{\mathrm{CC}}^{2}=\frac{\Gamma^{2}}{v} \frac{2}{3 \sqrt{2 \pi}} \sqrt{q}
$$

and

$$
\lim _{q \rightarrow \infty} w_{\mathrm{CD}}^{2}=0 \text { and } \lim _{u \rightarrow \infty} w_{\mathrm{CD}}^{2}=0
$$

In summary, the only non-zero contributions are (A.28) and (A.29).

\section{References}

[1] Edwards S F and Wilkinson D R 1982 Proc. R. Soc. A 381 17-31

[2] Barabási A L and Stanley H E 1995 Fractal concepts in surface growth (Cambridge, UK: Cambridge University Press)

[3] Täuber U C 2005 Critical dynamics preprint available at http://www.phys.vt.edu/ tauber/utaeuber.html, accessed 11 Feb 2010

[4] Le Bellac M 1991 Quantum and Statistical Field Theory [Phenomenes critiques aux champs de jauge, English] (New York, NY, USA: Oxford University Press) translated by G. Barton

[5] Pruessner G 2004 Phys. Rev. Lett. 92246101 (pages 4) (Preprint arXiv: cond-mat/0404007v2)

[6] Ma S K 1976 Modern Theory of critical Phenomena (Reading, MA, USA: Addison-Wesley)

[7] Privman V, Hohenberg P C and Aharony A 1991 Phase Transitions and Critical Phenomena vol 14 ed Domb C and Lebowitz J L (New York, NY, USA: Academic Press) chap 1, pp 1-134

[8] Krug J 1997 Adv. Phys. 46 139-282

[9] Meakin P 1998 Fractals, scaling and growth far from equilibrium (Cambridge, UK: Cambridge University Press) 
[10] Vicsek T 1999 Fractal Growth Phenomena 2nd ed (Singapore: World Scientific)

[11] Godrèche C (ed) 1992 Solids Far From Equilibrium (Cambridge, UK: Cambridge University Press)

[12] Family F and Vicsek T 1985 J. Phys. A: Math. Gen. 18 L75-L81

[13] Farkas Z and Fülöp T 2001 J. Phys. A: Math. Gen. 34 3191-3198

[14] Butkovskiy A G 1982 Green's Functions and Transfer Functions Handbook (Chichester, West Sussex, UK: Ellis Horwood Ltd.)

[15] Magnus W, Oberhettinger F and Soni R P 1966 Formulas and Theorems for the Special Functions of Mathematical Physics (Berlin, Germany: Springer-Verlag) 